Pacific

Journal of

Mathematics

AN EXTENSION OF WOLPERT'S DERIVATIVE FORMULA

\author{
Caroline Series
}

Volume 197 No. 1

January 2001 


\title{
AN EXTENSION OF WOLPERT'S DERIVATIVE FORMULA
}

\author{
Caroline Series
}

\begin{abstract}
A well known formula of Wolpert expresses the derivative of length of a geodesic $\gamma$ on a hyperbolic surface with respect to the Fenchel Nielsen twist about a fixed simple geodesic $C$ as a sum of the cosines of the intersection angles between the two curves. We derive an analogous formula for the derivative of the length of $\gamma$ with respect to variation of the length of the fixed curve $C$.
\end{abstract}

\section{Introduction.}

Let $\Sigma$ be an oriented hyperbolic surface and let $C$ be a simple closed geodesic on $\Sigma$. The Fenchel Nielsen twist about $C$ is a deformation of $\Sigma$ in which the surface is cut along $\Sigma$ and reglued after twisting one boundary curve distance $t$ relative to the other. Let $\gamma$ be a geodesic transverse to $C$, with hyperbolic length $l(\gamma)$; measured relative to a suitable base point, $l(\gamma)$ is a function of the twist parameter $t$. Wolpert [10], see also Kerckhoff [5], proved the following well known formula for the derivative with respect to $t$ :

$$
d l(\gamma) / d t=\Sigma_{j=1}^{k} \cos \theta_{j} .
$$

Here the sum is over the intersection points $Q_{j}$ of $C$ and $\gamma$, and $\theta_{j}$ is the angle, measured anticlockwise, from $\gamma$ to $C$ at $Q_{j}$.

The formula has been extended to a 3-dimensional situation by Kourouniotis [6]. Starting from a Fuchsian group uniformising the surface $\Sigma$, the Fenchel Nielsen twist along $C$ extends to a quakebend with the now complex parameter $t \in \mathbf{C}$, where $\Re t$ is the twist as above and $\Im t$ is the bending angle. This produces a family of deformations $\rho_{t}: \pi_{1}(\Sigma) \rightarrow P S L(2, \mathbf{C})$. The geodesic $\gamma$ on $\Sigma$ corresponds to a conjugacy class $[\gamma]$ in $\pi_{1}(\Sigma)$ and hence in $\rho_{t}\left(\pi_{1}(\Sigma)\right)$. The length $l(\gamma)$ extends to the complex translation length, also denoted $l(\gamma)$, of any element in the conjugacy class $\rho_{t}([\gamma])$. The derivative formula becomes

$$
d l(\gamma) / d t=\Sigma_{j=1}^{k} \cosh q_{j},
$$

where now $q_{j}$ is the complex distance (measured with suitable a convention on orientations) in $\mathbf{H}^{3}$ between a pair of axes $\tilde{C}(t)$ and $\tilde{\gamma}(t)$ of elements in 
the conjugacy classes of $\rho_{t}([C])$ and $\rho_{t}([\gamma])$, chosen so that the projections on $\Sigma$ of the time 0 lifts $\tilde{C}(0)$ and $\tilde{\gamma}(0)$ intersect in the point $Q_{j}$.

It is natural to ask for the analogy of Wolpert's formula for the derivative of $l(\gamma)$ with respect to the hyperbolic length $l=l(C)$ of the geodesic $C$. In this article we find such a formula and, more generally, find a similar expression for the derivative of $l(\gamma)$ with respect to $\zeta \in \mathbf{C}$, for any family of holomorphic deformations $\rho_{\zeta}: \pi_{1}(\Sigma) \rightarrow P S L(2, \mathbf{C})$. The formula is expressed in terms of the derivatives with respect to $\zeta$ of the complex lengths $l_{C}$ and the complex twist parameters $t_{C}$ of curves $C$ in a fixed pants decomposition of $\Sigma$. The parameters $\left(l_{C} / 2, t_{C}\right)$ are the complex Fenchel Nielsen coordinates for quasifuchsian space, as introduced by Kourouniotis [7] and Tan $[9]$.

To state the formula, we need a more detailed description of the trajectory of the geodesic $\gamma$ relative to a fixed pants decomposition of $\Sigma$. For simplicity, we shall describe the case of variation from a base point which is Fuchsian; the extension to the quasifuchsian setting is explained in 4.1. It is, however, convenient to state the formula in terms of the complex distance $\mathbf{d}(L, M)$ between oriented lines in $\mathbf{H}^{3}$. The details of our conventions are explained in Section 2.1 below.

Suppose then that we are given a fixed decomposition of $\Sigma$ into pairs of pants by a family of disjoint geodesics $\mathcal{C}$. The trajectory of the oriented geodesic $\gamma$ cuts in order a sequence $C_{i_{1}}, \ldots, C_{i_{k+1}}$ of pants curves $C_{i_{j}} \in \mathcal{C}$ in points $Q_{j}$, so that each adjacent pair $C_{i_{j}}, C_{i_{j+1}}$ are boundary curves of a unique pair of pants $\Pi_{j}$, numbered such that $Q_{k+1}=Q_{1}$ and such that the arc from $Q_{1}$ to $Q_{k+1}$ is exactly $\gamma$. The idea is that there is a unique curve homotopic to $\gamma$ with no backtracking, made up of arcs which wrap around a pants curve, alternating with arcs which cross the pants from one boundary to the next following the common perpendiculars between the boundaries.

More precisely, let $\tilde{\gamma}$ be the lift of $\gamma$ through a fixed lift $\tilde{Q}_{1}$ of $Q_{1}$. Then $\tilde{\gamma}$ intersects successively lifts $\tilde{C}_{j}$ of $C_{i_{j}}$ in points $\tilde{Q}_{j}$ which project to $Q_{j}$; in particular $\tilde{Q}_{k+1}$ is the image of $\tilde{Q}_{1}$ under the covering translation corresponding to $\tilde{\gamma}$.

Let $\tilde{D}_{j}$ denote the geodesic containing the common perpendicular to $\tilde{C}_{j}$ and $\tilde{C}_{j+1}$, oriented from the point $Q_{j}^{-}$where it meets $\tilde{C}_{j}$ to the point $Q_{j+1}^{+}$ where it meets $\tilde{C}_{j+1}$. Orient $\tilde{C}_{j}$ so that the angle from $\tilde{\gamma}$ to $\tilde{C}_{j}$ is anticlockwise (relative to a fixed given orientation on $\Sigma$ ). Clearly, $\gamma$ is freely homotopic to the projection on $\Sigma$ of the path made up of geodesic arcs from $Q_{j}^{-}$to $Q_{j}^{+}, Q_{j}^{+}$to $Q_{j+1}^{-}, j=1, \ldots, k$.

Define $d_{j}=\mathbf{d}\left(\tilde{C}_{j}, \tilde{C}_{j+1}\right)$ and $q_{j}=\mathbf{d}\left(\tilde{\gamma}, \tilde{C}_{j}\right)$. Define $s_{j}= \pm \mathbf{d}\left(\tilde{D}_{j}, \tilde{D}_{j+1}\right)$, where we choose the $+\operatorname{sign}$ if $Q_{j}^{-}$is to the left of $Q_{j}^{+}$on $\tilde{C}_{j}$ (relative to the orientation induced by the orientation of $\Sigma$ ), and the minus sign otherwise. Likewise, define $p_{j}= \pm \mathbf{d}\left(\tilde{\gamma}, \tilde{D}_{j}\right)$, where we choose the + sign if $\tilde{\gamma}$ intersects 
the geodesic extension of $\tilde{D}_{j}$ or if it does not intersect and the common perpendicular from $\tilde{\gamma}$ to $\tilde{D}_{j}$ is oriented in the same sense as $\tilde{C}_{j}$, and the sign otherwise. (In fact $d_{j}, s_{j}, p_{j}, q_{j}$ are really signed complex distances, that is, distances whose real parts are counted positive or negative depending on position relative to the orientation of the line along which they are measured, as explained in the next section. The signs of $p_{j}, q_{j}$ do not matter in the formula 3 below.)

The function $d_{j}$ depends only on the geometry of $\Pi_{j}$, see Section 3.1, and is holomorphic in the half lengths $l_{C} / 2$. The shift function $s_{j}$ is of the form $n_{j} l_{i_{j}} / 2+e_{j}+t_{i_{j}}$ where $n_{j} \in \mathbf{Z}, l_{i_{j}}$ is the length $l\left(C_{i_{j}}\right)$ of $C_{i_{j}}, t_{i_{j}}$ is the associated Fenchel Nielsen twist and $e_{j}$ satisfies $0 \leq \Re e_{j}<\Re l_{i_{j}} / 2$. The function $e_{j}$ depends only on the geometry of $\Pi_{j}$ and $\Pi_{j-1}$ and is again holomorphic in the parameters $l_{C} / 2$. The choices of $n_{j}, d_{j}, e_{j}$ are independent of the hyperbolic structure on $\Sigma$, and depend only on the topology of $\gamma$ relative to the pants decomposition $\mathcal{C}$. Details are explained in Section 4.2.

Letting ' denote derivative with respect to $\zeta$, our derivative formula is:

$$
l^{\prime}(\gamma)=\Sigma_{j=1}^{k} \cosh p_{j} d_{j}^{\prime}+\Sigma_{j=1}^{k} \cosh q_{j} s_{j}^{\prime} .
$$

In the special case in which the lengths $l\left(C_{i}\right)$, and hence the functions $d_{j}$ and $e_{j}$, are real valued and constant, we recover Wolpert's formula (1).

We give two proofs of Equation (3). The first is very similar to Kerckhoff's proof of (1) in [5] and the second is inspired by the proof of (1) in [2], in which $\rho_{t}(\gamma) \in S L(2, \mathbf{C})$ is expressed in terms of a product of twists associated to the distance $t_{C}$ quakebend along $C$. In the present case, we write $\rho_{\zeta}(\gamma)$ as a product of translations along the axes of the pants curves $\tilde{C}_{j}$ by the shift distances $s_{j}$, and translations along the common perpendiculars $\tilde{D}_{j}$ by distances $d_{j}$. None of these translations are elements in $\rho_{\zeta}\left(\pi_{1}(\Sigma)\right)$; nevertheless once $\rho_{\zeta}(\gamma)$ is expressed in this way, our formula drops out.

The plan of the paper is as follows. In Section 2 we discuss signed complex distance and review the trigonometry of right-angled hyperbolic hexagons, in particular obtaining a formula for the derivatives of the side lengths. In Section 3 we review hyperbolic pairs of pants and Fenchel Nielsen coordinates in both the two and three dimensional settings. In Section 4 we discuss how to keep track of geodesic paths and give careful definitions of the terms entering into (3). Finally in Section 5 we prove Equation (3) following the Kerckhoff method and in Section 6 give the version inspired by [2].

\section{Hyperbolic trigonometry.}

In this section, following [3], we express trigonometric formulae in terms of the signed complex distance between oriented geodesics in hyperbolic 3-space $\mathbf{H}^{3}$. Since the detailed conventions are important in our formulae, we give all the definitions here. 
2.1. Complex distance. The signed complex distance between oriented geodesics in hyperbolic 3 -space $\mathbf{H}^{3}$, relative to an oriented common perpendicular, should be thought of as the analogue of signed distance measured along an oriented axis in $\mathbf{H}^{2}$. It is defined as follows, see [3, V.3.].

Let $\alpha$ be any oriented line in $\mathbf{H}^{3}$, and let $P_{1}, P_{2} \in \alpha$. Let $d\left(P_{1}, P_{2}\right) \geq 0$ denote the hyperbolic distance between $P_{1}$ and $P_{2}$. We define the signed real hyperbolic distance $\delta_{\alpha}\left(P_{1}, P_{2}\right)$ as $d\left(P_{1}, P_{2}\right)$ if the orientation of the arc from $P_{1}$ to $P_{2}$ coincides with that of $\alpha$ and $-d\left(P_{1}, P_{2}\right)$ otherwise.

Now let $L_{1}, L_{2} \subset \mathbf{H}^{3}$ be oriented lines with distinct endpoints on the Riemann sphere $\hat{\mathbf{C}}$, with oriented common perpendicular $\alpha$ meeting $L_{1}, L_{2}$ in points $Q_{1}, Q_{2}$ respectively, where if $L_{1}, L_{2}$ intersect we take $Q_{1}=Q_{2}$. Let $\mathbf{v}_{\mathbf{i}}$ be tangent vectors to the positive directions of $L_{i}$ at $Q_{i}, i=1,2$. Let $\Pi$ be the hyperbolic plane through $Q_{2}$ orthogonal to $\alpha$ and let $\mathbf{w}_{\mathbf{1}}$ denote the parallel translate of $\mathbf{v}_{\mathbf{1}}$ along $\alpha$ to $Q_{2}$. Let $\mathbf{n}$ be a unit vector at $Q_{2}$ pointing in the positive direction $\alpha$. The signed complex distance between $L_{1}$ and $L_{2}$ is

$$
\boldsymbol{\delta}_{\mathbf{n}}\left(L_{1}, L_{2}\right)=\boldsymbol{\delta}_{\alpha}\left(L_{1}, L_{2}\right)=\delta_{\alpha}\left(Q_{1}, Q_{2}\right)+i \theta
$$

where $\theta$, measured modulo $2 \pi \mathbf{Z}$, is the angle between $\mathbf{w}_{\mathbf{1}}$ and $\mathbf{v}_{\mathbf{2}}$ measured anticlockwise in the plane spanned by $\mathbf{w}_{\mathbf{1}}, \mathbf{v}_{\mathbf{2}}$ and oriented by $\mathbf{n}$. Normalising so that $\alpha$ is the imaginary axis oriented from 0 to $\infty$ and $L_{1}, L_{2}$ are the oriented geodesics from -1 to 1 , and $-r e^{i \theta}$ to $r e^{i \theta}$ respectively $(r>0)$, we have $\boldsymbol{\delta}_{\alpha}\left(L_{1}, L_{2}\right)=\log r+i \theta$. Notice that the choice $\pm \theta$ is independent of whether or not $r>1$. In terms of cross ratio,

$$
\exp \boldsymbol{\delta}_{\alpha}\left(L_{1}, L_{2}\right)=\frac{\left(v_{2}-a^{-}\right)\left(v_{1}-a^{+}\right)}{\left(v_{2}-a^{+}\right)\left(v_{1}-a^{-}\right)}
$$

where $v_{1}, v_{2}$ are the positive endpoints of $L_{1}, L_{2}$ and $a^{-}, a^{+}$are respectively the negative and positive endpoints of $\alpha$ on $\hat{\mathbf{C}}$.

For any line oriented $L \subset \mathbf{H}^{3}$, denote by $L^{-1}$ the line $L$ with opposite orientation. We have $\boldsymbol{\delta}_{\alpha}\left(L_{1}, L_{2}\right)=-\boldsymbol{\delta}_{\alpha}\left(L_{2}, L_{1}\right), \boldsymbol{\delta}_{\alpha^{-1}}\left(L_{1}, L_{2}\right)=-\boldsymbol{\delta}_{\alpha}\left(L_{1}, L_{2}\right)$ and $\boldsymbol{\delta}_{\alpha}\left(L_{1}^{-1}, L_{2}\right)=\boldsymbol{\delta}_{\alpha}\left(L_{1}, L_{2}^{-1}\right)=\boldsymbol{\delta}_{\alpha}\left(L_{1}, L_{2}\right)+i \pi$.

Finally we define the (unsigned) complex distance $\mathbf{d}\left(L_{1}, L_{2}\right)$ as $\mathbf{d}\left(L_{1}, L_{2}\right)$ $=\boldsymbol{\delta}_{\alpha}\left(L_{1}, L_{2}\right)$ if $\Re \delta_{\alpha}\left(L_{1}, L_{2}\right)>0,-\delta_{\alpha}\left(L_{1}, L_{2}\right)$ if $\Re \delta_{\alpha}\left(L_{1}, L_{2}\right)<0$ and $i\left|\delta_{\alpha}\left(L_{1}, L_{2}\right)\right|$ otherwise. The relations for unsigned distance become $\mathbf{d}\left(L_{1}, L_{2}\right)$ $=\mathbf{d}\left(L_{2}, L_{1}\right)$, and $\mathbf{d}\left(L_{1}^{-1}, L_{2}\right)=\mathbf{d}\left(L_{1}, L_{2}^{-1}\right)=\mathbf{d}\left(L_{1}, L_{2}\right)+i \pi$. When $L_{1}$ and $L_{2}$ have a common endpoint $Q$, we define $\mathbf{d}\left(L_{1}, L_{2}\right)=0$ if the orientations of $L_{1}$ and $L_{2}$ at $Q$ are the same and $\mathbf{d}\left(L_{1}, L_{2}\right)=i \pi$ otherwise.

Suppose that $L$ and $M$ lie in a common plane $\Pi$, oriented by a normal vector $\mathbf{n}$. If $L$ intersects $M$ at an angle $\theta$ measured anticlockwise from $L$ to $M$, then $\boldsymbol{\delta}_{\mathbf{n}}(L, M)=i \theta$, while if $L$ and $M$ are disjoint then $\boldsymbol{\delta}_{\mathbf{m}}(L, M)=$ $p+\epsilon i \pi$, where $p$ is the unsigned perpendicular distance between $L$ and $M$, $\mathbf{m}$ is a vector oriented from $L$ to $M$ and $\epsilon=0$ if $L$ and $M$ have the same orientation relative to their common perpendicular and $\epsilon=1$ otherwise. 
Finally, suppose that $M \in P S L(2, \mathbf{C})$ is non-parabolic with axis Ax $M$, oriented pointing from the repelling to the attracting fixed point. We define the complex length $l(M)$ as $\boldsymbol{\delta}_{\mathrm{Ax} M}(L, M(L))$, where $L$ is any oriented line perpendicular to Ax $M$. Notice that with this definition, $\Re l(M)>0$ so that in fact $l(M)=\mathbf{d}(L, M(L))$. If $M$ is parabolic, we define $l(M)=0$.

2.1.1. Signed versus unsigned complex distance. With the above definitions, the signed complex distance between $L_{1}$ and $L_{2}$ depends on the orientation of their common perpendicular, while the (unsigned) complex distance does not. Both signed and unsigned distance take values in $\mathbf{C} / 2 \pi i \mathbf{Z}$. Notice that one cannot express the signed complex distance in terms of the endpoints of $L_{1}, L_{2}$ alone, since any possible formula will fail to see the orientation of the common perpendicular $\alpha$. In fact, as can easily be checked by normalising as above,

$$
\cosh \boldsymbol{\delta}_{\alpha}\left(L_{1}, L_{2}\right)=\cosh \mathbf{d}\left(L_{1}, L_{2}\right)=\frac{1+\chi}{1-\chi}
$$

where $\chi=\frac{\left(u_{1}-u_{2}\right)\left(v_{1}-v_{2}\right)}{\left(u_{1}-v_{2}\right)\left(v_{1}-u_{2}\right)}$ and $u_{i}, v_{i}$ are respectively the repelling and attracting fixed points of $L_{i}$. In this formula, the distinction between signed and unsigned complex distance is lost.

The formulae of hyperbolic trigonometry are often given in terms of unsigned complex distance, see for example in [8] or [7]. However, it will be important for us to use signed complex distance for the following reason. If $L_{1}$ and $L_{2}$ vary so as to move through an intersection point changing their positions relative to the orientation of their common perpendicular $\alpha$, then there is a discontinuity in $\mathbf{d}\left(L_{1}, L_{2}\right)$. Since we want to vary the positions of all our lines and to obtain a formula that is holomorphic in the relevant parameters, we have to use not the complex distance $\mathbf{d}\left(L_{1}, L_{2}\right)$, but the holomorphic variable $\boldsymbol{\delta}_{\alpha}\left(L_{1}, L_{2}\right)$ associated to some pre-specified orientation of $\alpha$. See also [4] for a similar use.

2.2. Right-angled hexagons. An oriented skew right-angled hyperbolic hexagon is a cyclically ordered set of six oriented geodesics $L_{n}$ in $\mathbf{H}^{3}$ indexed by $n \in \mathbf{Z}(\bmod 6)$, such that $L_{n}$ intersects $L_{n+1}$ orthogonally. (We do not require all the lines $L_{n}$ to be distinct, nor do we require them to be oriented consistently around the hexagon.) Define $\sigma_{n}=\boldsymbol{\delta}_{\hat{L}_{n}}\left(\hat{L}_{n-1}, \hat{L}_{n+1}\right)$. The hyperbolic sine and cosine rules, [3] VI.2, are:

Sine Rule.

$$
\frac{\sinh \sigma_{1}}{\sinh \sigma_{4}}=\frac{\sinh \sigma_{3}}{\sinh \sigma_{6}}=\frac{\sinh \sigma_{5}}{\sinh \sigma_{2}}
$$

Cosine Rule.

$$
\cosh \sigma_{n}=\frac{\cosh \sigma_{n+3}-\cosh \sigma_{n+1} \cosh \sigma_{n-1}}{\sinh \sigma_{n+1} \sinh \sigma_{n-1}} .
$$


The definitions also extend to include degenerate hexagons in which the line $L_{n}$ has shrunk to a point, or more precisely in which $L_{n-1}$ and $L_{n+1}$ have a common end point at infinity which we call $L_{n}$. In this case, the real parts of both $\sigma_{n-1}$ and $\sigma_{n+1}$ are infinite and $\sigma_{n}=i \pi$. The cosine formula still holds for the side length $\sigma_{n+3}$ in this case. If the hexagon is planar with sides of real length $l_{n}, n \bmod 6$, and we orient the sides consistently around the hexagon, then $\sigma_{i}=l_{i}+i \pi$ so that $\cosh \sigma_{i}=-\cosh l_{i}, \sinh \sigma_{i}=-\sinh l_{i}$ and we recover the classical cosine formula, see for example [1, p. 148].

We remark that the cosine rule proves the well-known fact $[\mathbf{3}, \mathrm{VI} .4]$ that the signed (complex) lengths of three alternate sides of a skew hexagon determine the other three, uniquely up to simultaneous reversal of orientation of the three other sides. We shall use later a discussion in terms of unsigned length from [7] Proposition 1.6: given any three $\sigma_{i} \in \mathbf{C}$ with $\Re \sigma_{i}>0$, there are two choices of triple $\left(\hat{\sigma}_{1}, \hat{\sigma}_{2}, \hat{\sigma}_{3}\right), \hat{\sigma}_{i} \in\left\{\sigma_{i}, \sigma_{i}+i \pi\right\}$ for which there is an skew hexagon with sides oriented consistently around the hexagon whose alternate sides have complex lengths $\hat{\sigma}_{i}$. These two hexagons are unique up to isometry and agree up to reversing orientations. Other choices of $\hat{\sigma}_{i}$ give the same hexagon but with different orientation of sides.

We shall need a formula for the complex distance between opposite sides of a skew hexagon. With the notation above, let $M_{n}$ be the common perpendicular to $L_{n}, L_{n+3}$ and let $p_{n}=\mathbf{d}\left(L_{n}, L_{n+3}\right)$.

Lemma 2.1. With the above notation,

$$
\cosh p_{n}=-\sinh \sigma_{n-2} \sinh \sigma_{n-1} .
$$

(Notice that this formula does not depend on the orientation of $M_{n}$.)

Proof. This is just the pentagon formula [3, VI.2 (2)]. It may be derived from the cosine formula as follows. Choose an orientation for $M_{n}$, and adjoin an extra oriented side $L$ to the pentagon formed by the lines $L_{n}, M_{n}, L_{n+3}, L_{n-2}, L_{n-1}$ through the intersection point of $L_{n-1}$ and $L_{n}$. We find $\boldsymbol{\delta}_{L}\left(L_{n-1}, L_{n}\right)= \pm i \pi / 2$ and $\boldsymbol{\delta}_{L_{n-1}}\left(L_{n-2}, L\right)=\sigma_{n-1} \pm i \pi / 2$. Substituting into the cosine formula for $\cosh \boldsymbol{\delta}_{L_{n-1}}\left(L_{n-2}, L\right)$ gives the result.

We shall also need the following:

Lemma 2.2. In a skew right-angled hexagon with complex side lengths $\sigma_{n}$ : $\cosh \sigma_{n-1} \sinh \sigma_{n+1}+\sinh \sigma_{n-1} \cosh \sigma_{n+1} \cosh \sigma_{n}=-\cosh \sigma_{n+2} \sinh \sigma_{n+3}$ $\sinh \sigma_{n-1} \cosh \sigma_{n+1}+\cosh \sigma_{n-1} \sinh \sigma_{n+1} \cosh \sigma_{n}=-\cosh \sigma_{n-2} \sinh \sigma_{n-3}$.

Proof. To prove the first formula, start from the cosine formula (5) with $\cosh \sigma_{n+2}$ on the left hand side and substitute $\cosh \sigma_{n} \sinh \sigma_{n-1} \sinh \sigma_{n+1}+$ $\cosh \sigma_{n-1} \cosh \sigma_{n+1}$ for $\cosh \sigma_{n+3}$. The other equation is similar. 
Remark. The trigonometrical formulae given for example in [8] and [7] refer to hexagons in which the sides are oriented consistently around the hexagon, and the complex distances between sides are measured unsigned (i.e., $\Re \sigma_{i}>0$ ). This is correct, since clearly in this case, signed and unsigned distances coincide. More generally, it is easy to verify that the sine and cosine formulae are unchanged after changing orientation of one of the sides. Thus by successively changing orientations one can always arrive a hexagon in which the sides are consistently oriented, to which the formulae for unsigned distance with $\Re \sigma_{i}>0$ apply.

\subsection{The derivative formula in a hexagon.}

Proposition 2.3. Suppose that, with the notation of Section 2.2, we are given a skew right-angled hexagon with oriented sides and that the signed complex distances $\sigma_{n}$ depend holomorphically on some variable $\zeta \in \mathbf{C}$. Let' denote derivative with respect to $\zeta$. For $n \bmod 6$, let $p_{n, n+3}=\mathbf{d}\left(L_{n}, L_{n+3}\right)$. Then

$$
\cosh p_{n, n+3} \sigma_{n}^{\prime}=-\left(\sigma_{n+3}^{\prime}+\cosh \sigma_{n-2} \sigma_{n-1}^{\prime}+\cosh \sigma_{n+2} \sigma_{n+1}^{\prime}\right) .
$$

Proof. Start with the cosine formula (5) in the form

$$
\sinh \sigma_{n+1} \sinh \sigma_{n-1} \cosh \sigma_{n}=\cosh \sigma_{n+3}-\cosh \sigma_{n+1} \cosh \sigma_{n-1} .
$$

Differentiating with respect to $\zeta$ and using Lemma 2.2 one obtains

$$
\begin{aligned}
& \sinh \sigma_{n+1} \sinh \sigma_{n-1} \sinh \sigma_{n} \sigma_{n}^{\prime} \\
& =\sinh \sigma_{n+3}\left(\sigma_{n+3}^{\prime}+\cosh \sigma_{n+2} \sigma_{n-1}^{\prime}+\cosh \sigma_{n-2} \sigma_{n+1}^{\prime}\right) .
\end{aligned}
$$

Applying the sine rule (4) and Lemma 2.1 gives the result.

\section{Pants and Fenchel Nielsen coordinates.}

3.1. Pants and Skew Pants. A (planar) hyperbolic pair of pants $\Pi$ is a three holed sphere with geodesic boundary curves $C_{i}$ of lengths $l_{i}>0, i=$ $1,2,3$. We also allow the degenerate case in which one or more $l_{i}$ vanish and the corresponding boundary curve is a puncture.

There is a unique common perpendicular, of length $a_{k}$, between each pair of distinct boundary curves $C_{i}, C_{j}$. If $C_{k}$ is a puncture, then $a_{i}$ and $a_{j}$ have infinite length. Cutting along the three common perpendiculars dissects $\Pi$ into two right-angled hexagons. Since the three common perpendiculars form three alternate sides of each hexagon, the two hexagons are congruent and the points at which the perpendiculars from $C_{j}$ and $C_{i}$ meet $C_{k}$ bisect $C_{k}$. Thus $\Pi$ is determined up to isometry by the lengths $l_{i}$ of the curves $C_{i}$; conversely, given any $l_{i} \geq 0, i=1,2,3$, there is a unique pair of pants for which $l\left(C_{i}\right)=l_{i}$. 
There is also a common perpendicular from $C_{k}$ to itself. This arc does not in general bisect $C_{k}$, but it divides $C_{k}$ into two arcs which are bisected by the endpoints on $C_{k}$ of the common perpendiculars from $C_{i}$ and $C_{j}$. Let $2 b_{k, i}, 2 b_{k, j}$ be the hyperbolic lengths of the arcs on $C_{k}$ containing the endpoints of the perpendiculars from $C_{i}, C_{j}$ respectively, so that $2\left(b_{k, i}+b_{k, j}\right)=l_{k}$. All distances $a_{i}, b_{k, i}$ and their derivatives can be computed from the hexagon formulae. For $a_{i}$ we use the hexagons described above while for $b_{k, i}$ we use a hexagon obtained by cutting $\Pi$ along the common perpendiculars from $C_{k}$ to itself and from $C_{i}$ to $C_{k}$. For example, in the special case in which $l_{k}$ only varies we find from the derivative formula (6):

$$
a_{i}^{\prime}=\frac{-l_{k}^{\prime}}{2 \tanh a_{j} \sinh l_{i} / 2}, a_{k}^{\prime}=\frac{-l_{k}^{\prime}}{2 \sinh a_{j} \sinh l_{i} / 2}, b_{k, i}^{\prime}=\frac{-\sinh b_{k, i} a_{i}^{\prime}}{\tanh a_{k} / 2} .
$$

(These formulae refer to the real hyperbolic distances: complex distances are obtained by consistently orienting the sides of the hexagons and replacing $l_{k}$ by $l_{k}+i \pi$ etc.) Notice that the complex versions of the variables $a_{k}, b_{i, k}$ are actually holomorphic functions of the half lengths $l_{i} / 2$.

3.1.1. Example. In the special case of a once punctured torus, we need to consider a degenerate hexagon $H$ whose three alternate side have lengths $l_{C} / 2, l_{C} / 2,0$. The only one of the above parameters to consider is the length $a_{C}$ of the common perpendicular to the two sides of lengths $l_{C} / 2$. This is given by the formula $\sinh l_{C} / 2 \sinh a_{C} / 2=1$, obtained by applying the cosine formula in $H$. Either differentiating this directly (and using the alternative form $\cosh l_{C} / 2 \tanh a_{C} / 2=1$ ), or as a limiting case of Equation (6) as the length of one side goes to zero, we find $a_{C}^{\prime}=-\left(\sinh l_{C} / 2\right)^{-1} l_{C}^{\prime}$.

3.2. Fenchel Nielsen coordinates. Let $S$ be a fixed oriented topological surface of negative Euler characteristic, possibly with punctures. A pants decomposition of $S$ is a collection $\mathcal{C}=\left\{C_{i}\right\}$ of disjoint simple closed curves which cut it into three holed spheres $\mathcal{P}=\left\{\Pi_{j}\right\}$, where we allow that some of the holes may be punctures. The curve $C$ either represents a loop round a puncture, or is a boundary component of two pants $\Pi^{\prime}, \Pi^{\prime \prime} \in \mathcal{P}$, where possibly $\Pi^{\prime}=\Pi^{\prime \prime}$. In the second case, fix once and for all a free homotopy class of oriented curves $K_{C}$ on $\Pi^{\prime} \cup \Pi^{\prime \prime}$ as follows: If $\Pi^{\prime} \neq \Pi^{\prime \prime}$ then $K_{C}$ intersects $C$ twice and separates $\Pi^{\prime} \cup \Pi^{\prime \prime} \cup C$, while if $\Pi^{\prime}=\Pi^{\prime \prime}$ then $K_{C}$ intersects $C$ once and does not separate $\Pi^{\prime} \cup C$.

Suppose that $\psi: S \rightarrow \Sigma$ is a homeomorphism from $S$ to a hyperbolic surface $\Sigma$. There is a unique smooth geodesic in each free homotopy class of non-boundary parallel curves on $S$. The lengths $l_{C}$ of the geodesics $C \in \mathcal{C}$ determine hyperbolic structures on the pants $\Pi_{j}$ up to isometry. The length $l_{C}$ is zero if and only if $C$ represents a puncture. For each curve $C \in \mathcal{C}$ with $l_{C}>0$, we define gluing data $t_{C}$ as follows. Suppose first that $\Pi^{\prime} \neq \Pi^{\prime \prime}$. As described in the introduction, a lift $\tilde{K}_{C}$ of the geodesic representing $K_{C}$ will 
cut three distinct lifts $\tilde{C}_{1}, \tilde{C}_{2}, \tilde{C}_{3}$ in succession in points $Q_{1}, Q_{2}, Q_{3}$ say, where $Q_{3}$ is the image of $Q_{1}$ under the covering translation of $\mathbf{H}^{2}$ corresponding to $K_{C}$. As in the introduction, orient $\tilde{C}_{2}$ so that the angle from $\tilde{K}_{C}$ to $\tilde{C}_{2}$ is anticlockwise, relative to the given orientation on $S$. With the notation of the introduction with $\tilde{\gamma}=\tilde{K}_{C}$, define $t_{C}=s_{2}=\boldsymbol{\delta}_{\tilde{C}_{i}}\left(\tilde{D}_{j-1}, \tilde{D}_{j}\right)$, where the common perpendicular $\tilde{D}_{j}$ is oriented from $\tilde{C}_{j}$ to $\tilde{C}_{j+1}$. In the case $\Pi^{\prime}=\Pi^{\prime \prime}$ the definition is the same except that we only need to consider two lifts $\tilde{C}_{1}, \tilde{C}_{2}$ of $C$.

Notice that if $t_{C}=0$, then the geodesic $K_{C}$ is orthogonal to $C$. In this case, we note that if $\Pi^{\prime}=\Pi^{\prime \prime}$ then the common perpendicular from $C$ to itself is the smooth closed geodesic $K_{C}$, while if $\Pi^{\prime} \neq \Pi^{\prime \prime}$ then $K_{C}$ is exactly the union of the common perpendiculars to $C$ in $\Pi^{\prime}$ and $\Pi^{\prime \prime}$.

Fixing a base surface $\Sigma_{0}$ for which $t_{C}=0, C \in \mathcal{C}$, the parameters $\left(l_{C}, t_{C}\right), C \in \mathcal{C}, l_{C} \in \mathbf{R}^{+}, t_{C} \in \mathbf{R}$ are the classical Fenchel Nielsen coordinates for the Teichmüller space of $S$ relative to $\mathcal{C}$ and $\Sigma_{0}$. Punctures are represented by curves $C$ with $l_{C}=0$. For these curves, the twist $t_{C}$ is undefined. Notice that since $t_{C}$ is defined as a signed distance, it defines a real analytic function on $\operatorname{Teich}(S)$.

\subsection{Skew pants, complex Fenchel Nielsen coordinates and quasi-} fuchsian groups. It is shown in [7] and [9] that the above construction for pairs of pants can be extended to $\mathbf{H}^{3}$. We summarize their construction here.

As noted in 2.2, any triple $\sigma_{1}, \sigma_{2}, \sigma_{3} \in \mathbf{C}$ with $\Re \sigma_{i}>0$ determines a pair of oriented skew right-angled hexagons which are isometric but with opposite orientations, and whose alternate sides have complex lengths $\hat{\sigma}_{1}, \hat{\sigma}_{2}, \hat{\sigma}_{3}$ for some choice of $\hat{\sigma}_{i} \in\left\{\sigma_{i}, \sigma_{i}+i \pi\right\}$. Gluing these two oppositely oriented hexagons gives a skew pair of pants whose boundary curves have lengths $2 \sigma_{i}$. Conversely, suppose we want to construct skew pants whose boundary curves have lengths $l_{1}, l_{2}, l_{3} \in \mathbf{C}$ with $\Re l_{i}>0$. This means that we need skew hexagons whose sides have complex lengths $l_{i} / 2$ or $l_{i} / 2+i \pi$. There is a unique pair of choices which gives a pair of oppositely oriented skew hexagons. Similar formulae to those of Section 3 are obtained for the complex lengths of the common perpendiculars to the boundaries of the pants. The construction extends to the case in which some or all of the three loxodromics are replaced by parabolics, in which case the axis is replaced by the fixed point at infinity, and the skew hexagons degenerate to pentagons with one vertex at infinity and two infinite sides.

A similar choice occurs when we try to represent $\pi_{1}(\Pi)$ in $S L(2, \mathbf{C})$. Suppose $M \in P S L(2, \mathbf{C})$ has complex translation length $l(M)$. Then $\operatorname{Tr} M$ is only defined up to multiplication by \pm 1 and $l(M) / 2$ is only defined up to addition of $i \pi$. These ambiguities are reflected in the formula $\operatorname{Tr} M=$ $2 \cosh l(M) / 2$. If $\Pi$ is a pair of pants, then the fundamental group $\pi_{1}(\Pi)$ 
is generated by the boundary loops $c_{1}, c_{2}, c_{3}$ which can be chosen such that $c_{1} c_{2} c_{3}=\mathrm{id}$. The following proposition shows that in fact, a representation of $\pi_{1}(\Pi)$ into $S L(2, \mathbf{C})$ is determined up to conjugation by a choice of half lengths $l_{i} / 2$.

Proposition 3.1 ([7, Proposition 2.3]). Let $\sigma_{1}, \sigma_{2}, \sigma_{3} \in \mathbf{C}$ with $\Re \sigma_{i}>0$. Then there is a homomorphism $\pi_{1}(\Pi) \rightarrow S L(2, \mathbf{C})$ such that $\operatorname{Tr}\left(\rho\left(c_{i}\right)\right)=$ $-2 \cosh \sigma_{i}, i=1,2,3$. This homomorphism is unique up to conjugation in $S L(2, \mathbf{C})$, provided that the endpoints of the axes $\rho\left(c_{i}\right)$ are pairwise distinct.

We note that the condition that the endpoints of the axes $\rho\left(c_{i}\right)$ be distinct holds if the group $\rho$ is the restriction to $\pi_{1}(\Pi)$ of a quasifuchsian representation of $\pi_{1}(S)$.

Suppose now that $S$ is a fixed oriented topological surface with pants decomposition $\mathcal{C}$ as above. Suppose that we are given parameters $l_{C} / 2, t_{C} \in \mathbf{C}$ with $\Re l_{C} \geq 0$ and $\Re l_{C}=0$ if and only if $C$ represents a puncture. Let $\Pi^{\prime}, \Pi^{\prime \prime} \in \mathcal{P}$ be two pants which meet along the curve $C$. Suppose for definiteness that $\Pi^{\prime} \neq \Pi^{\prime \prime}$. We want to glue these pants along the curve $C$, using the complex gluing date $t_{C}$. Use the half lengths and Proposition 3.1 to construct a representation for $\pi_{1}\left(\Pi^{\prime}\right) \rightarrow S L(2, \mathbf{C})$. Following the notation of Section 3.2, we see that, up to orientation preserving isometry in $\mathbf{H}^{3}$, this fixes $\tilde{C}_{1}$ and $\tilde{C}_{2}$; it also fixes the sign of the trace of the element corresponding to $C$. Let $\tilde{D}_{1}$ be the common perpendicular from $\tilde{C}_{1}$ to $\tilde{C}_{2}$, with the orientation inherited from its orientation on the base surface $\Sigma_{0}$. Choose a representation of $\pi_{1}\left(\Pi^{\prime \prime}\right)$ in which the image of $C$ is the axis $\tilde{C}_{2}$. This fixes the axis $\tilde{C}_{3}$ and the common perpendicular $\tilde{D}_{1}$ from $\tilde{C}_{2}$ to $\tilde{C}_{3}$ up to a translation and rotation about $\tilde{C}_{2}$. Moreover, if the parameter $l_{C} / 2$ is given, then by Proposition 3.1 the signs of the trace of the element corresponding to $C$ in these two representations agree. Thus a unique representation of $\pi_{1}\left(\Pi^{\prime} \cup \Pi^{\prime \prime}\right)$ is specified by requiring $t_{C}=\boldsymbol{\delta}_{\tilde{C}_{2}}\left(D_{1}, D_{2}\right)$. With the notation of the introduction, $t_{C}$ is exactly the shift parameter $s_{2}$ so that $\Re t_{C}$ is the real twist, while $\Im t_{C}$ is the bending angle between the skew pants along their common boundary $C$. Successively gluing all the pants in this way determines a representation $\rho: \pi_{1}(S) \rightarrow S L(2, \mathbf{C})$, unique up to conjugation in $S L(2, \mathbf{C})$, in which $\operatorname{Tr} \rho(C)=-\cosh L_{C} / 2$ and $\rho(C)$ is a loxodromic with complex length $l_{C}$.

If the image $\rho\left(\pi_{1}(S)\right)$ is discrete in $S L(2, \mathbf{C})$ then $H^{3} / \rho\left(\pi_{1}(S)\right)$ is a hyperbolic 3-manifold; if $H^{3} / \rho\left(\pi_{1}(S)\right) \approx S \times(0,1)$ then $\rho\left(\pi_{1}(S)\right)$ is quasifuchsian: this is the case in a neighbourhood of Fuchsian groups for which the image is contained up to conjugation in $S L(2, \mathbf{R})$. Quasifuchsian space $\mathcal{Q F}(S)$ is the space of representations $\rho\left(\pi_{1}(S)\right) \rightarrow P S L(2, \mathbf{C})$ for which $\rho\left(\pi_{1}(S)\right)$ is quasifuchsian, modulo conjugation in $\operatorname{PSL}(2, \mathbf{C})$. The parameters $l_{C} / 2, t_{C}$ are holomorphic parameters for $\mathcal{Q F}(S)$; they are the the complex Fenchel Nielsen coordinates introduced in [7] and [9]. 


\section{Geodesic paths.}

4.1. Intersection points. To make sense of formula (3), we need to keep track of axes and of intersection points between geodesics as we vary the representation $\rho: \pi_{1}(S) \rightarrow P S L(2, \mathbf{C})$ in $\mathcal{Q F}(S)$. This we do as follows. Suppose first $\rho\left(\pi_{1}(S)\right)$ is Fuchsian, uniformising a hyperbolic surface $\Sigma$, and let $\Sigma_{0}$ be a fixed base surface as above. The natural homeomorphism $\rho$ : $\Sigma_{0} \rightarrow \Sigma$ lifts to a homeomorphism of $\mathbf{H}^{2}$ which extends to a homeomorphism $h$ of the boundary $S^{1}$. Let $\alpha=\alpha\left(\Sigma_{0}\right)$ be any geodesic, not necessarily closed, on $\Sigma_{0}$. Let $\tilde{\alpha}=\tilde{\alpha}\left(\Sigma_{0}\right)$ be a lift of $\alpha$ to $\mathbf{H}^{2}$ with endpoints $a, b$ on $S^{1}$. These endpoints map to the endpoints $h(a), h(b)$ of a corresponding geodesic $\tilde{\alpha}(\Sigma)$ which projects to a geodesic $\alpha(\Sigma)$ on $\Sigma$. Now suppose $\alpha\left(\Sigma_{0}\right), \beta\left(\Sigma_{0}\right)$ are two such geodesics which meet at a point $Q=Q\left(\Sigma_{0}\right) \in \Sigma_{0}$. Lift $Q$ to a point $\tilde{Q} \in \mathbf{H}^{2}$ and choose lifts $\tilde{\alpha}\left(\Sigma_{0}\right), \tilde{\beta}\left(\Sigma_{0}\right)$ of $\alpha, \beta$ through $\tilde{Q}$. We define $\tilde{Q}(\Sigma)$ to be the intersection of $\tilde{\alpha}(\Sigma), \tilde{\beta}(\Sigma)$ and $Q(\Sigma)$ to be its projection on $\Sigma$.

As a special case, let $\gamma_{i} \in \pi_{1}(S), i=1,2$ and let $\Gamma_{0}$ and $\Gamma$ be the Fuchsian groups uniformising $\Sigma_{0}$ and $\Sigma$ respectively. Denoting by $\gamma_{i}(\Sigma)$ the unique smooth geodesic on $\Sigma$ in the class $\gamma_{i}$, we note that since $h$ conjugates the actions of $\Gamma_{0}$ and $\Gamma$ on $S^{1}$, this construction defines a bijection between the intersection points of $\gamma_{1}\left(\Sigma_{0}\right)$ and $\gamma_{2}\left(\Sigma_{0}\right)$ on $\Sigma_{0}$, and $\gamma_{1}(\Sigma)$ and $\gamma_{2}(\Sigma)$ on $\Sigma$.

In the case of quasifuchsian groups, we can keep track of intersections in a similar way. Let $\Gamma=\rho(S)$ be quasifuchsian so that we are given an isomorphism $\Gamma_{0} \rightarrow \Gamma$. The boundary $S^{1}$ of $\mathbf{H}^{2}$ is replaced by the limit set $\Lambda$ of $\Gamma$; there is a natural homeomorphism $S^{1} \rightarrow \Lambda$ which conjugates the actions of $\Gamma_{0}$ and $\Gamma$. The endpoints of a lift of $\tilde{\alpha}\left(\Sigma_{0}\right)$ map to the endpoints of a geodesic in $\tilde{\alpha}(\Gamma)$ in $\Lambda$. In this case, the geodesics $\tilde{\alpha}(\Gamma), \tilde{\beta}(\Gamma)$ do not in general intersect, and we replace $\tilde{Q}(\Gamma)$ with the common perpendicular between $\tilde{\alpha}(\Gamma)$ and $\tilde{\beta}(\Gamma)$, oriented with the orientation inherited from $\Sigma_{0}$.

4.2. Geodesic paths and the terms in formula (3). We are finally able to give precise definitions of all terms in our formula (3). As above, suppose that $S$ is a fixed oriented surface with a pants decomposition $\mathcal{C}=\left\{C_{i}\right\}$. We need to describe a free homotopy class $\gamma$ on $S$ in terms of the topology of $S$ and the decomposition $\mathcal{C}$. We do this by describing the trajectory of the oriented geodesic representing $\gamma$ on the base surface $\Sigma_{0}$, relative to the geodesic representatives $C=C\left(\Sigma_{0}\right)$ of the curves in $\mathcal{C}$ and their common perpendiculars in the pants in $\mathcal{P}$.

As in the introduction, define the sequences of oriented geodesics $\tilde{C}_{j}\left(\Sigma_{0}\right)$, $\tilde{D}_{j}\left(\Sigma_{0}\right)$ and of points $\tilde{Q}_{j}\left(\Sigma_{0}\right), Q_{j}^{-}\left(\Sigma_{0}\right), Q_{j}^{+}\left(\Sigma_{0}\right)$ relative to the surface $\Sigma_{0}$. (Notice we mean by $\tilde{D}_{j}$ the complete geodesic in $\mathbf{H}$, not just the arc from $\tilde{C}_{j}$ to $\tilde{C}_{j+1}$.) If we vary $\Sigma_{0}$ by changing either the length parameters $l_{C}$ or the twists $t_{C}$, we can keep track of lifts of geodesics as in 4.1. Since all the curves in question are defined by their endpoints on $S^{1}$, we see that the 
corresponding sequence for the trajectory of a lift $\tilde{\gamma}(\Sigma)$ is just the sequence $\tilde{C}_{j}(\Sigma), \tilde{D}_{j}(\Sigma)$ with corresponding intersection points $\tilde{Q}_{j}(\Sigma), Q_{j}^{-}(\Sigma), Q_{j}^{+}(\Sigma)$. As explained in Section 4.1, we can still keep track of lifts even in the quasifuchsian case.

Let $N_{j}$ be the oriented common perpendicular to $\tilde{\gamma}$ and $\tilde{C}_{j}(\Sigma)$, oriented consistently with the orientation on $\Sigma_{0}$. We define $q_{j}=\boldsymbol{\delta}_{N_{j}}\left(\tilde{\gamma}, \tilde{C}_{j}\right)$. Thus in the planar case, $q_{j}=i \theta_{i}$ where $\theta_{i}$ is the anticlockwise angle from $\tilde{\gamma}$ to $\tilde{C}_{j}$.

The geodesics $\tilde{\gamma}$ and $\tilde{D}_{j}(\Sigma)$ may or may not intersect. However, as explained in Section 4.1, they intersect if and only if they intersect in the case of the base surface $\Sigma_{0}$. Let $F_{j}$ be the common perpendicular to $\tilde{\gamma}$ and $\tilde{D}_{j}(\Sigma)$, oriented consistently with the orientation on $\Sigma_{0}$ if $\tilde{\gamma} \cap \tilde{D}_{j} \neq \emptyset$, and consistently with $\tilde{C}_{j}$ and $\tilde{C}_{j+1}$ relative to $\tilde{\gamma}$ otherwise. We define $p_{j}=\boldsymbol{\delta}_{F_{j}}\left(\tilde{\gamma}, \tilde{D}_{j}\right)$, coinciding with the definition of $p_{i}$ given in the introduction in the planar case.

The arc of $\tilde{D}_{j}(\Sigma)$ between $Q_{j}^{-}(\Sigma), Q_{j+1}^{+}(\Sigma)$ projects to the common perpendicular between the boundary components $C_{i_{j}}(\Sigma)$ and $C_{i_{j+1}}(\Sigma)$ of $\Pi_{j}$. We define $d_{j}=\boldsymbol{\delta}_{\tilde{D}_{j}}\left(\tilde{C}_{j}, \tilde{C}_{j+1}\right)$. We note that in the planar case, $\boldsymbol{\delta}_{\tilde{D}_{j}}\left(\tilde{C}_{j}, \tilde{C}_{j+1}\right)$ $=\mathbf{d}\left(\tilde{C}_{j}, \tilde{C}_{j+1}\right)$. Hence in this case $d_{j}$ can be computed in terms of the half lengths $l_{C} / 2, C \in \mathcal{C}$ : it is the complex distance $a_{j}+i \pi$ corresponding to one of the lengths $a_{j}$ discussed in Section 3.1. In general, the function $d_{j}$ is the analytic continuation to the case in which the parameters $l_{C} / 2$ become complex.

Now consider the shift functions $s_{j}$ defined as $s_{j}=\boldsymbol{\delta}_{\tilde{C}_{j}}\left(\tilde{D}_{j-1}, \tilde{D}_{j}\right)$. (Notice that in the planar case this definition coincides with the one given in the introduction.) Clearly from the definitions, $s_{j}\left(l_{C} / 2, t_{C}\right)=s_{j}\left(l_{C} / 2,0\right)+t_{i_{j}}$, where $s_{j}\left(l_{C} / 2, t_{C}\right)$ denotes the parameter in the group with complex Fenchel Nielsen coordinates $\left(l_{C} / 2, t_{C}\right), C \in \mathcal{C}$. The group with parameters $\left(l_{C} / 2,0\right)$ is chosen so that on each axis $\tilde{C}_{j}$, the endpoints of certain of the common perpendiculars between two boundary components of $\Pi_{j-1}, \Pi_{j}$ coincide. Thus $s_{j}\left(l_{C} / 2,0\right)=n_{j} l_{i_{j}}\left(l_{C} / 2,0\right) / 2+e_{j}\left(l_{C} / 2,0\right)$ where $n_{j} \in \mathbf{Z}$ and $0 \leq \Re e_{j}<\Re l_{i_{j}} / 2$.

Finally, we consider the possible values of $e_{j}\left(l_{C} / 2,0\right)$. By definition the pants $\Pi_{j-1}, \Pi_{j}$ which meet along $C_{j}$ are glued (for the group with coordinates $\left.\left(l_{C} / 2,0\right)\right)$ in such a way that $K_{C}\left(\Sigma\left(l_{C} / 2,0\right)\right)$ is smooth. The points $Q_{j}^{-}(\Sigma), Q_{j}^{+}(\Sigma)$ project to endpoints of the common perpendiculars between two boundary components of $\Pi_{j-1}, \Pi_{j}$ respectively. Thus if $\tilde{D}_{j-1}, \tilde{D}_{j}$ project to the common perpendiculars from $C_{j}$ to itself in $\Pi_{j-1}, \Pi_{j}$ respectively, then $e_{j}=0$. Otherwise, $\tilde{D}_{j-1}, \tilde{D}_{j}$ project to others among the common perpendiculars and it is clear that $e_{j}$ can take on one of at most a small number of values of the form $x b+y b^{\prime}$ where $x, y \in\{0, \pm 1\}$ and $b=b_{k, i}, b^{\prime}=b_{k^{\prime}, i^{\prime}}$ are as 
defined in Section 3.1 relative to the pants $\Pi_{j-1}$ and $\Pi_{j}$ and the boundary curve $C_{j}$.

4.2.1. Example. Continuing our example in which $S$ is a once punctured torus, any simple loop $C$ cuts $S$ into a pair of pants whose boundary components consist of two holes and one puncture. Since no loops on $S$ pass through the puncture, any loop $\gamma$ is homotopic to a loop made up of arcs which wrap around $C$ and arcs which follow the common perpendicular from from $C$ to itself. Formula (3) becomes

$$
l^{\prime}(\gamma)=a_{C}^{\prime} \Sigma_{j=1}^{k} \cosh p_{j}+\Sigma_{j=1}^{k}\left(m_{j} l_{C}^{\prime}+t_{C}^{\prime}\right) \cosh q_{j}
$$

where $m_{j} \in \mathbf{Z}$ is the number of times $\gamma$ wraps around $C$ at the $j^{\text {th }}$ intersection and $a_{C}^{\prime}=\left(\sinh l_{C} / 2\right)^{-1} l_{C}^{\prime}$.

\section{Proof of the derivative formula: Method 1 .}

In our first proof of the derivative formula (3), we use a method similar to that of Kerckhoff in [5]. We continue with the notation of Section 4 for the lift $\tilde{\gamma}$ of a trajectory of an oriented geodesic $\gamma$.

Let $F_{j}$ be the oriented common perpendicular to $\tilde{\gamma}$ and $\tilde{D}_{j}$, as defined in Section 4.2 above. Consider the right-angled hexagon $H_{j}$ with oriented sides

$$
\tilde{\gamma}, F_{j}, \tilde{D}_{j}, \tilde{C}_{j}, \tilde{D}_{j-1}, F_{j-1}
$$

(The sides here are not oriented consistently.) Let $g_{j}=\boldsymbol{\delta}_{\tilde{\gamma}}\left(F_{j-1}, F_{j}\right), u_{j}=$ $\boldsymbol{\delta}_{\tilde{D}_{j-1}}\left(F_{j-1}, \tilde{C}_{j}\right), v_{j}=\boldsymbol{\delta}_{\tilde{D}_{j}}\left(\tilde{C}_{j}, F_{j}\right)$. Notice that $d_{j-1}=v_{j-1}+u_{j}$, and that $\sum_{j=1}^{k} g_{j}=l(\gamma)+2 k \pi, k \in \mathbf{Z}$

Taking careful account of order to determine the signs, we obtain from the formula (6):

$$
-\cosh q_{j} s_{j}^{\prime}=-\left(g_{j}^{\prime}-\cosh p_{j-1} u_{j}^{\prime}-\cosh p_{j} v_{j}^{\prime}\right) .
$$

Substituting $d_{j-1}^{\prime}-v_{j-1}^{\prime}$ for $u_{j}^{\prime}$ and summing over $j$ gives the result.

We remark that in the formula (3), the interpretation of the quantities $p_{j}, q_{j}$ as signed (as opposed to unsigned) complex distances is unimportant since the terms $\cosh p_{j}, \cosh q_{j}$ do not depend on whether the complex distance is signed or not. In case of $d_{j}$, in the planar case we automatically have $d_{j}>0$. The only situation in which one would have to consider signed distance is if the group were deformed through representations $\rho\left(\pi_{1}(S)\right)$ for which the axes $\tilde{C}_{j}, \tilde{C}_{j+1}$ intersect. While this can certainly happen for arbitrary representations $\rho$ (for example, choose $\sigma_{1} \in \mathbf{R}^{+}+i \pi, \sigma_{2}, \sigma_{3} \in \mathbf{R}^{+}$in Proposition 3.1, see the explicit formula for $\rho$ in [7]), we have not been able to determine whether or not it can occur for $\rho\left(\pi_{1}(S)\right) \in \mathcal{Q F}(S)$. 


\section{Proof of the derivative formula: Method 2.}

In this version of the proof, we use a method suggested by the computation of derivative with respect to a quakebend given in [2, Section 3.9]. The main point is to express the element representing $\rho_{\zeta}(\gamma) \in P S L(2, \mathbf{C})$ as a product of translations along the axes $\tilde{C}_{j}, \tilde{D}_{j}$. There are two reasons to give this proof, at first sight more complicated than that in method 1 . Firstly, it sheds rather more light on the meaning of individual terms in the formula, and secondly, it illustrates a point which the author believes to be important, that it may often be useful to express a group element as a product of "elementary" transformations each of which has a simple geometrical meaning, but which may however not be in the group.

If $L$ is any oriented line in $\mathbf{H}^{3}$ and $\xi \in \mathbf{C}, \Re \xi>0$, then we denote by $\Delta(\xi, L) \in P S L(2, \mathbf{C})$ the unique loxodromic with axis $L$, complex length $\xi$, and attracting fixed point the positive endpoint of $L$. If $\Re \xi<0$, then by definition $\Delta(\xi, L)$ has axis $L$, complex length $-\xi$, and attracting fixed point the negative endpoint of $L$. In both cases, $\operatorname{Tr} \Delta(\xi, L)=2 \cosh (\xi / 2)$, where the ambiguity in the sign of the trace is the same as the ambiguity of $i \pi$ in the choice of $\xi / 2$.

Proposition 6.1. With the notation of the introduction,

$$
\Delta\left(l(\gamma), \tilde{\gamma}_{1}\right)=\Delta\left(d_{k}, \tilde{D}_{k}\right) \Delta\left(s_{k}, \tilde{C}_{k}\right) \ldots \Delta\left(d_{1}, \tilde{D}_{1}\right) \Delta\left(s_{1}, \tilde{C}_{1}\right)
$$

and

$$
\Delta\left(l(\gamma), \tilde{\gamma}_{1}\right)=\Delta\left(s_{1}, \tilde{C}_{k+1}\right) \Delta\left(d_{k}, \tilde{D}_{k}\right) \ldots \Delta\left(s_{2}, \tilde{C}_{2}\right) \Delta\left(d_{1}, \tilde{D}_{1}\right),
$$

where $\tilde{\gamma}_{1}$ is the lift of $\gamma$ which intersects $\tilde{C}_{1}$ in $\tilde{Q}_{1}$.

Proof. Denote the product on the right hand side of the first equation by $T$. We have to see that $T$ maps $\tilde{\gamma}_{1}$ to itself and maps $\tilde{Q}_{1}=\tilde{\gamma}_{1} \cap \tilde{C}_{1}$ to $\tilde{Q}_{k+1}=\tilde{\gamma}_{1} \cap \tilde{C}_{k+1}$. It is clearly enough to show that $T\left(\tilde{C}_{1}\right)=\tilde{C}_{k+1}$ and $T\left(\tilde{Q}_{1}\right)=\tilde{Q}_{k+1}$. Now $\Delta\left(s_{r}, \tilde{C}_{r}\right)$ maps $\tilde{C}_{r}$ to itself and $\tilde{Q}_{r}^{-}$to $\tilde{Q}_{r}^{+}$while $\Delta\left(d_{r}, \tilde{D}_{r}\right)$ maps $\tilde{C}_{r}$ to $\tilde{C}_{r+1}$ and $\tilde{Q}_{r}^{+}$to $\tilde{Q}_{r+1}^{-}$. Thus $T=\tilde{\gamma}_{1}$; the proof of the other expression is similar.

Now fix once and for all axes $I, J$ which intersect at right angles in a fixed point $P$. For a finite set $\left\{z_{1}, \ldots, z_{r}\right\}, z_{j} \in \mathbf{C}$, write $\left\{z_{1}, \ldots, \hat{z}_{i}, \ldots, z_{r}\right\}$ for $\left\{z_{1}, \ldots, z_{r}\right\} \backslash\left\{z_{i}\right\}$. The following are obvious consequences of the construction in the above proof.

Corollary 6.2. For $i=1, \ldots, k$, let $\tilde{\gamma}_{i}$ be the lift of $\gamma$ which intersects $\tilde{C}_{i}$ at $Q_{i}$. Then there are elements $E_{i}, U_{i} \in P S L(2, \mathbf{C})$ such that $E_{i}\left(Q_{i}^{-}\right)=$ $P, E_{i}\left(\tilde{C}_{i}\right)=I, E_{i}\left(\tilde{D}_{i}\right)=J, E_{i} \tilde{\gamma}_{i} E_{i}^{-1}=U_{i} \Delta\left(s_{i} ; I\right)$ and such that $U_{i}$ depends only on $\left\{d_{1}, s_{1}, \ldots, \hat{s}_{i}, \ldots, s_{k}\right\}$. Likewise there are elements $F_{i}, V_{i} \in$ 
$\operatorname{PSL}(2, \mathbf{C})$ such that $F_{i}\left(Q_{i}^{+}\right)=P, F_{i}\left(\tilde{C}_{i}\right)=I, F_{i}\left(\tilde{D}_{i}\right)=J, F_{i} \tilde{\gamma}_{i} F_{i}^{-1}=$ $V_{i} \Delta\left(d_{i} ; J\right)$ and such that $V_{i}$ depends only on $\left\{d_{1}, s_{1}, \ldots, \hat{d}_{i}, \ldots, s_{k}\right\}$.

Corollary 6.3. Let $L$ be any oriented line in $\mathbf{H}^{3}$. Then $\operatorname{Tr}(\Delta(l(\gamma), L))=$ $f\left(d_{1}, s_{1}, \ldots, d_{k}, s_{k}\right)$, where the function $f$ depends only on the variables $\left\{d_{1}, s_{1}, \ldots, d_{k}, s_{k}\right\}$.

Now suppose that the coordinates $l_{C} / 2, \tau_{C}, C \in \mathcal{C}$ depend holomorphically on a complex variable $\zeta$. As in Section 2.2, the $d_{i}$ are holomorphic functions of the half lengths $l_{C} / 2$. Thus the function $f$ is holomorphic in $\zeta$. To compute $d l(\gamma) / d \zeta$, first note that for any $M=M(\zeta) \in S L(2, \mathbf{C})$ for which $l(M) / 2$ varies holomorphically with respect to $\zeta$, we have $d \operatorname{Tr}(M(\zeta)) / d \zeta=$ $\sinh (l(M) / 2) d l(M) / d \zeta$. From Corollary 6.3 we have

$$
\frac{d \operatorname{Tr}(\Gamma(\zeta))}{d \zeta}=\sum_{i} \frac{\partial f}{\partial s_{i}} s_{i}^{\prime}+\sum_{i} \frac{\partial f}{\partial d_{i}} d_{i}^{\prime} .
$$

Thus using Corollary 6.2, we have only to evaluate $\partial \operatorname{Tr}\left(U_{i} \Delta\left(s_{i} ; I\right)\right) / \partial s_{i}$ and $\partial \operatorname{Tr}\left(V_{i} \Delta\left(d_{i} ; J\right)\right) / \partial d_{i}$. This can be done by essentially the same computation as that given in [2, Section 3.9]. Here is an alternative coordinate-free proof using our formula (6). As usual, if $M \in P S L(2, \mathbf{C})$ is loxodromic, Ax $M$ denotes the axis of $M$ oriented from the repelling to the attracting fixed point.

Lemma 6.4. Let $L$ be an oriented line in $\mathbf{H}^{3}$, and let $B \in P S L(2, \mathbf{C})$ be fixed. Let $A(\eta)=\Delta(\eta ; L)$. Then

$$
\frac{d(l(B A(\eta)))}{d \eta}=-\cosh \mathbf{d}\left(L, \operatorname{Ax}(B A(\eta))^{\epsilon}\right) \quad \epsilon \in\{ \pm 1\},
$$

where the choice of $\epsilon$ is made in such a way that the lines $L, \operatorname{Ax}(B A(\eta))^{\epsilon}$, $\mathrm{Ax} B^{\epsilon^{\prime}}$ are alternate sides in a consistently oriented skew hexagon for suitable $\epsilon^{\prime} \in\{ \pm 1\}$.

Proof. Given $A, B \in P S L(2, \mathbf{C})$ one can as in Section 2.2 define a skew hexagon whose alternate sides are the axes of $A, B$ and $B A$ with lengths $l(A) / 2, l(B) / 2, l(B A) / 2$, where the ambiguities of $i \pi$ depend on the choice of orientation of the axes. (This construction works even in the degenerate case in which $\mathrm{Ax} A$ and $\mathrm{Ax} B$ coincide; the hexagon degenerates to a line but the cosine formula is still valid.) Make this construction with $A(\eta)$ and $B$, orienting all sides consistently with the orientation of $L=$ $\operatorname{Ax} A(\eta)$. Let $\rho(\eta)=\mathbf{d}\left(L, \operatorname{Ax}(B A(\eta))^{\epsilon}\right)$. We note that from the definitions, $\mathbf{d}(\operatorname{Ax} A(\eta), \operatorname{Ax} B)=\mathbf{d}\left(L, \operatorname{Ax} B^{\epsilon^{\prime}}\right)$ is constant. The result is obtained by applying formula (6) for the derivative of this last expression, and noting that derivatives are independent of the above ambiguities of $i \pi$. 
To complete the proof of Equation (3), we note that using the expression $E_{i} \tilde{\gamma}_{i} E_{i}^{-1}=U_{i} \Delta\left(s_{i} ; I\right)$ from Corollary 6.2 , since $U_{i}$ does not depend on $s_{i}$,

$$
\frac{\partial f}{\partial s_{i}}=-\sinh (l(\gamma) / 2) \cosh \mathbf{d}\left(I, \operatorname{Ax}\left(E_{i} \tilde{\gamma}_{i} E_{i}^{-1}\right)^{\epsilon_{i}}\right) \quad \epsilon_{i} \in\{ \pm 1\}
$$

and

$$
\mathbf{d}\left(I, \operatorname{Ax} E_{i} \tilde{\gamma}_{i} E_{i}^{-1}\right)=\mathbf{d}\left(E_{i}^{-1}(I), \operatorname{Ax} \gamma_{i}^{\epsilon_{i}}\right)=\mathbf{d}\left(\tilde{C}_{i}, \operatorname{Ax} \gamma_{i}^{\epsilon_{i}}\right) .
$$

We need to decide on the choice of orientation of $\epsilon_{i}$. One way to do this is to argue by direct computation as in [2]. Alternatively, it is not hard to see in the planar case that the attracting and repelling fixed points of the axes of $A, B, B A$ occur in the anticlockwise order

$$
B^{-},(B A)^{-}, A^{-}, B^{+},(B A)^{+}, A^{+}
$$

round the circle at infinity, where $M^{+}$and $M^{-}$denote the attracting and repelling fixed points of $M \in P S L(2, \mathbf{C})$ respectively. Thus to make an oriented skew hexagon whose alternate sides are these three axes, the orientation of $\operatorname{Ax} B A$ must be reversed. This shows that in the planar case, $\epsilon_{i}=-1$ and $\mathbf{d}\left(\tilde{C}_{i}, \operatorname{Ax} \gamma_{i}^{-1}\right)=q_{i}+i \pi$. The result now follows using the similar formula for $\frac{\partial f}{\partial d_{i}}$ and cancelling a factor of $\sinh (l(\gamma) / 2)$ from both sides.

\section{References}

[1] A.F. Beardon, The Geometry of Discrete Groups, Springer-Verlag, New York, 1983.

[2] D.B.A. Epstein and A. Marden, Convex hulls in hyperbolic space, a theorem of Sullivan, and measured pleated surfaces, In D.B.A. Epstein, editor, Analytical and Geometric Aspects of Hyperbolic Space, LMS Lecture Notes, 111, 112-253, Cambridge University Press, 1987.

[3] W. Fenchel, Elementary Geometry in Hyperbolic Space, de Gruyter, 1989.

[4] D. Gabai, G.R. Meyerhoff and N. Thurston, Homotopy Hyperbolic 3-Manifolds are Hyperbolic, MSRI preprint series no. 1996-058, 1996.

[5] S. Kerckhoff, The Nielsen realization problem, Annals Math., 117 (1983), 235-265.

[6] C. Kourouniotis, The geometry of bending quasi-Fuchsian groups, LMS Lecture Notes, 173, 148-164, Cambridge University Press, 1992.

[7] C. Kourouniotis, Complex length coordinates for quasifuchsian groups, Mathematika, 41 (1994), 173-188.

[8] J.R. Parker and C. Series, Bending formulae for convex hull boundaries, J. d'Analyse Math., 67 (1995), 165-198.

[9] S.P. Tan, Complex Fenchel-Nielsen coordinates for quasifuchsian structures, Int'l. J. Math., 5(2) (1994), 239-251. 
[10] S. Wolpert, An elementary formula for the Fenchel-Nielsen twist, Comment. Math. Helvetici, 56 (1981), 132-135.

Received December 14, 1998.

\author{
Mathematics Institute \\ WARWICK UNIVERSITY \\ COVENTRY CV4 7AL \\ UNITED KINGDOM \\ E-mail address: cms@maths.warwick.ac.uk
}

